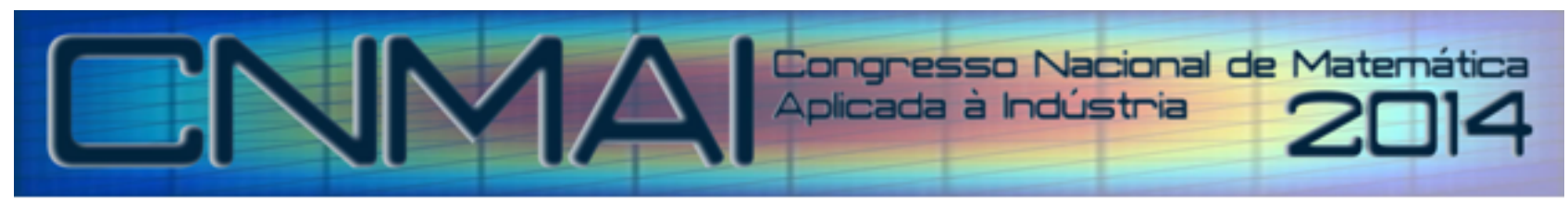

18 a 21 de novembro de 2014, Caldas Novas - Goiás

\title{
PROBLEMA INTEGRADO DE DIMENSIONAMENTO DE LOTES E CORTE DE ESTOQUE COM RESTRIÇÃO DE CICLOS DA SERRA
}

\author{
Gislaine M. Melega, gislainemelega@gmail .com ${ }^{1}$ \\ Matheus Vanzela, matheusvanzela@gmail. com ${ }^{1}$ \\ Silvio A. de Araujo, saraujo@ibilce.unesp.br.tres ${ }^{1}$ \\ Socorro Rangel, socorro@ibilce. unesp.br. quatro ${ }^{1}$ \\ ${ }^{1}$ Unesp - Ibilce, Departamento de Matemática Aplicada, São José do Rio Preto, SP
}

\begin{abstract}
Resumo. Neste trabalho aborda-se o problema integrado de dimensionamento de lotes e corte de estoque. O objetivo do problema integrado é capturar a interdependência entre as decisões destes problemas, possibilitando economia de matéria prima e diminuição dos gastos no processo de produção. Um modelo da literatura é discutido, no qual os autores realizam um estudo de caso em uma fábrica de móveis de pequeno porte e consideram em seu modelo, restrições para o controle do número de ciclos da serra. Propomos uma extensão deste conjunto de restrições que resulta em um modelo não linear. Uma estratégia de linearização é aplicada a estas restrições, obtendo assim um modelo linear. Pretende-se com este novo modelo, estender a técnica de geração de colunas a qual foi aplicada ao modelo original e discutir os resultados obtidos.
\end{abstract}

Palavras-chave: Problema de Dimensionamento de Lotes, Problema de Corte de Estoque, Problema Integrado, Ciclos da Serra.

\section{INTRODUÇÃO}

Estudar o problema de dimensionamento de lotes $(P D L)$ de forma integrada ao problema de corte de estoque $(P C E)$ constitui uma área de relevância na literatura devido a sua grande aplicabilidade no setor industrial. O objetivo do problema integrado é capturar a interdependência entre as decisões, de modo a possibilitar economia de matéria-prima e diminuição dos custos globais no processo de produção. Assim, o problema integrado de dimensionamento de lotes e corte de estoque consiste em decidir, em cada período do horizonte de planejamento a quantidade de produtos finais (itens) a serem produzidos, de maneira a minimizar o custo total. No custo total são incluídos custos de preparo e estocagem referentes ao problemas de dimensionamento de lotes e a quantidade de objetos a serem cortados, este último, objetivo do problema de corte de estoque.

Diversos trabalhos da literatura tratam o $P D L$ e o $P C E$ de maneira integrada em diferentes setores industriais, entre eles Farley (1988) em uma fábrica de roupas (corte bidimensional), Hendry et al. (1996) em uma fábrica de cobre (corte unidimensional), Nonas e Thorstenson (2008) em uma fábrica de caminhões off-road e Poltroniere et al. (2008) em uma indústria de papel. No setor moveleiro, destacamos os trabalhos de Gramani e França (2006), Gramani et al. (2011), Ghidini e Arenales (2009), Santos et al. (2011), Alem e Morabito (2012) e Vanzela et al. (2013).

Em Vanzela et al. (2013), o problema integrado é estudado no contexto do processo produtivo de uma fábrica de móveis de pequeno porte. Um aspecto importante no modelo apresentado, é a restrição de capacidade da máquina de corte (serra), em que se possibilita o corte simultâneo de vários objetos. O conjunto de todas as operações necessárias para cortar um, ou mais objetos simultaneamente, de acordo com um determinado padrão de corte é definido como ciclo da serra (Yanasse et al., 1991). No entanto, Vanzela et al. (2013) consideraram na restrição de ciclos da serra que padrões de corte diferentes podem compartilhar o mesmo ciclo da serra, o que consiste em uma relaxação para situações encontradas na prática de um ambiente industrial.

Como objetivo deste trabalho, propomos uma reformulação para a restrição referente ao ciclo da serra, na qual considera-se que cada ciclo da serra seja composto por um único padrão de corte. O modelo reformulado constitui um modelo de programação não linear, para o qual aplica-se uma estratégia de linearização baseada em abordagens encontradas na literatura. Exemplos numéricos são apresentados para validar o novo modelo. Resultados preliminares deste trabalho são discutidos em Melega et al. (2014). 


\section{DESCRIÇÃO DO PROBLEMA}

A indústria de móveis no Brasil está concentrada em pólos regionais localizados principalmente nas regiões Sul e Sudeste do país. Diversos tipos de matéria-prima são usados na produção de móveis (madeira, metal, plástico, couro). Os pólos da região noroeste do estado de São Paulo são voltados principalmente para a produção de móveis residenciais de madeira (Figueiredo e Rangel, 2008), no qual encontra-se a fabrica deste estudo, aqui denotada por Fábrica L.

A Fábrica L, situada no pólo de Votuporanga, é uma empresa característica do setor e considerada de pequeno porte em função do número de funcionários. A empresa ocupa-se da produção de móveis residenciais de madeira, retilíneos, na sua maioria móveis para dormitório. Para representar o processo de tomada de decisão da Fábrica L, Vanzela et al. (2013) fizeram algumas simplificações no processo produtivo.

\section{Simplificações}

1. Somente a capacidade do setor de corte é considerada e presume-se que os demais setores podem lidar com as decisões tomadas para o problema de corte de estoque.

2. O tempo de preparo para a máquina de corte e o tempo de troca de padrão de corte não foi levado em consideração. O impacto estimado desta simplificação é levado em consideração indiretamente na capacidade da máquina de corte.

3. O limite de capacidade de produção em termos do número total de ciclos da serra em um dado período é relaxado, de forma que diferentes padrões de corte podem ser cortados no mesmo ciclo de serra.

4. Atraso na entrega, e horas extras para atender a demanda não são permitidos.

5. As cores dos produtos finais não são considerados.

O problema tratado consiste então em definir o dimensionamento dos lotes de móveis (produtos finais) e o número de objetos a serem cortados (e os respectivos padrões de corte) de forma a minimizar o custo total de produção considerando restrições relativas ao atendimento da demanda dos itens finais e à capacidade da máquina de corte. Neste trabalho a demanda é considerada determinística e a capacidade da máquina de corte é definida em termos do número máximo de ciclos da serra em cada período.

\section{MODELAGEM MATEMÁTICA}

Nesta seção é apresentado o modelo matemático proposto por Vanzela et al. (2013) para o problema integrado no contexto de fabricação de móveis. Para tanto, faz-se o uso dos seguintes índices e dados.

\section{Índices:}

$t=1, \ldots, T:$ periodos;

$f=1, \ldots, F:$ produtos finais;

$p=1, \ldots, P$ : peças;

$e=1, \ldots, E$ : espessura da placa;

$j=1, \ldots, J:$ padrões de corte.

\section{Parâmetros:}

$c_{f}$ : $\quad$ custo de produção para o produto $f$;

$h_{f}: \quad$ custo de estoque para o produto $f$;

$D_{f t}$ : demanda por produto $f$ no período $t$;

$o^{e}$ : $\quad$ espessura da placa $e$;

$\mathrm{co}^{e}: \quad$ custo da placa com espessura $e$;

$\widehat{h}_{p}^{e}$ : $\quad$ custo de estoque da peça $p$ com espessura $e$;

$C_{t}$ : $\quad$ capacidade máxima de produção no período $t$, contabilizada em número de ciclos da serra;

$q_{p f}^{e}$ : número de peças $p$ de espessura $e$ necessárias para produzir uma unidade do produto $f$;

$S: \quad$ altura da serra;

$\operatorname{cap}^{e}: \quad$ número máximo de placas de espessura $e$ que podem ser cortadas simultaneamente $\left(\left\lfloor\frac{S}{o^{e}}\right\rfloor\right)$;

$a_{p j}^{e}: \quad$ número de peças $p$ com espessura $e$ presentes no padrão de corte $j$;

$I_{f 0}: \quad$ estoque inicial do produto final $f$;

$I P_{p 0}^{e}: \quad$ estoque inicial da peça $p$ com espessura $e$

ts : $\quad$ porcentagem da demanda utilizada para impor níveis seguros de estoque dos produtos finais;

\section{Variáveis:}

$X_{f t}: \quad$ quantidade do produto $f$ produzida no período $t$;

$I_{f t}: \quad$ quantidade de produto $f$ armazenada no final do período $t$,

$I P_{p t}^{e}: \quad$ número de peças $p$ de espessura $e$ armazenadas em período de $t$,

$y_{j t}^{e}: \quad$ número de placas de espessura $e$, cortadas de acordo com o padrão $j$ no período $t$. 
Formulação do Problema: PIDCM

$$
\min Z=\sum_{f=1}^{F} \sum_{t=1}^{T}\left(c_{f} X_{f t}+h_{f} I_{f t}\right)+\sum_{e=1}^{E} \sum_{j=1}^{J} \sum_{t=1}^{T} c o^{e} y_{j t}^{e}+\sum_{e=1}^{E} \sum_{p=1}^{P} \sum_{t=1}^{T} \widehat{h}_{p}^{e} I P_{p t}^{e}
$$

Sujeito a:

$$
\begin{aligned}
& X_{f t}+I_{f, t-1}-I_{f t}=D_{f t}, \\
& I_{f t} \geqslant t s D_{f t}, \\
& I_{f T} \geqslant t s\left(\sum_{t=1}^{T} D_{f t}\right) \\
& f=1, \ldots, F ; t=1, \ldots, T \\
& f=1, \ldots, F ; t=1, \ldots, T-1 \\
& \sum_{j=1}^{J} a_{p j}^{e} y_{j t}^{e}+I P_{p, t-1}^{e}-I P_{p t}^{e}=\sum_{f=1}^{F} q_{p f}^{e} X_{f t} \\
& f=1, \ldots, F \\
& p=1, \ldots, P ; t=1, \ldots, T ; e=1, \ldots, E \\
& \sum_{e=1}^{E} \sum_{j=1}^{J} \frac{y_{j t}^{e}}{\text { cap }^{e}} \leqslant C_{t} \\
& y_{j t}^{e} \in \mathbb{Z}_{+} \text {, } \\
& X_{f t}, I_{f t} \in \mathbb{R}_{+} \text {, } \\
& I P_{p t}^{e} \in \mathbb{R}_{+} \text {, } \\
& t=1, \ldots, T \\
& j=1, \ldots, J ; t=1, \ldots, T ; e=1, \ldots, E \\
& f=1, \ldots, F ; t=1, \ldots, T \\
& p=1, \ldots, P ; t=1, \ldots, T ; e=1, \ldots, E
\end{aligned}
$$

A função objetivo (1) minimiza a soma dos custos de produção $\left(c_{f}\right)$, estoque final dos produtos $\left(h_{f}\right)$, custos de matéria-prima $\left(c o^{e}\right)$ e os custos de estocar as peças $\left(\widehat{h}_{p}^{e}\right)$. As restrições (2) garantem que a demanda dos produtos finais $f$ seja atendida. As restrições (3) e (4) impõem níveis de estoque de segurança para o produto final $f$ como uma porcentagem da demanda de cada produto. Para os primeiros $(t-1)$ períodos, os níveis de segurança são expressos em termos das demandas individuais $\left(t s D_{f t}\right)$, e para o período final, os níveis de estoque de segurança são expressos em termos da demanda total. O conjunto de restrições (5) modela a interdependência entre o $P D L$ e o $P C E$, pois considera as decisões relativas ao dimensionamento de lotes $\left(X_{f t}\right)$ e a decisão sobre o corte de matéria-prima $\left(y_{j t}^{e}\right)$. O estoque de peças $\left(I P_{p t}^{e}\right)$ é permitido. As restrições (6) garantem o respeito à capacidade de produção $\left(C_{t}\right)$ definida em número de ciclos da serra em cada período $t$. As restrições (7), (8) e (9) definem domínio das variáveis.

Na simplificação 3 proposta por Vanzela et al. (2013) referente à capacidade da serra, padrões de corte distintos podem compartilhar o mesmo ciclo da serra. Este restrição foi assim considerada, com o objetivo de evitar que a solução do problema de corte de estoque seja inviável na prática. Para tratar esta simplificação, propomos uma reformulação para a restrição de capacidade da serra, que será descrita na próxima seção.

\section{PROPOSTA DE REFORMULAÇÃO}

Nesta seção será apresentada uma reformulação para a restrição referente ao ciclo da serra do problema integrado (restrição 6). Alguns trabalhos na literatura abordam problemas que possuem em sua estrutura o ciclo da serra. Yanasse et al. (1991) desenvolveram uma heurística para resolver o PCE bidimensional com o objetivo de reduzir o número de ciclos da serra. Posteriormente Mosquera e Rangel (2007) propuseram 2 variações da heurística apresentada por Yanasse et al. (1991) para um estudo de caso em uma indústria moveleira. Yanasse (2008) mostra que a solução do problema de corte de estoque considerando a redução de ciclos da serra em uma situação de alta demanda é equivalente a resolver o $P C E$ com as demandas escaladas pelo número máximo de objetos que a máquina pode cortar ao mesmo tempo. Ranck JR. (2008) propõe um modelo matemático para o problema de corte de estoque que considera a redução do número total de ciclos da serra e o número total de objetos a serem cortados, que pode ser usado tanto no caso unidimensional como no bidimensional, e permite também o uso de ciclos incompletos.

Antes de apresentar a reformulação, faz-se necessário a seguinte definição.

Definição 1. (Toscano et al., 2013): O número mínimo de ciclos da serra, necessários para cortar yje objetos de espessura e, de acordo com um determinado padrão de corte $j$ e com a capacidade da serra é calculado de acordo com:

$$
\left\lceil\frac{y_{j}^{e}}{\operatorname{cap}^{e}}\right\rceil \quad j=1, \ldots, J ; e=1, \ldots, E
$$

A fim de tratar situações encontradas na prática industrial, este trabalho reformula a restrição de ciclo da serra (restrição 6 do modelo PIDCM), de modo que seja considerado apenas um único tipo de padrão de corte em cada ciclo da serra. Primeiramente, isto é feito ao substituir $\frac{y_{j t}^{e}}{c a p^{e}}$ por $\left\lceil\frac{y_{j t}^{e}}{c a p^{e}}\right]$ em (6), obtendo uma reformulação para a restrição de ciclos da serra, expressa pela restrição (11) (Melega et al., 2014). Ao efetuar esta mudança, o número de ciclos é contado exatamente e uma restrição mais forte é obtida, já que uma solução que era factível para a restrição (6) pode agora ser infactível. 


$$
\sum_{e=1}^{E} \sum_{j=1}^{J}\left\lceil\frac{y_{j t}^{e}}{c^{e} p^{e}}\right\rceil \leq C_{t}, \quad t=1, \ldots, T
$$

Uma desvantagem que surge ao considerar esta nova restrição no modelo, é o fato de torná-lo não linear. Para tratar esta dificuldade, uma estratégia de linearização foi aplicada na restrição (11) e é baseada em Yanasse (2008) e Mosquera e Rangel (2007). Esta estratégia consiste em definir uma nova variável $z_{j t}^{e}$ que contabiliza a quantidade de ciclos da serra utilizados pra cortar o objeto de espessura $e$ de acordo com o padrão de corte $j$ no período $t$. Deste modo, a restrição (11) é linearizada através das seguintes restrições (12) - (14):

$$
\begin{array}{lr}
\frac{y_{j t}^{e}}{c a p^{e}} \leq z_{j t}^{e} & j=1, \ldots, J ; t=1, \ldots, T ; e=1, \ldots, E \\
\sum_{e=1}^{E} \sum_{j=1}^{J} z_{j t}^{e} \leqslant C_{t} & t=1, \ldots, T ; \\
z_{j t}^{e} \in \mathbb{Z}_{+}, & j=1, \ldots, J ; t=1, \ldots, T ; e=1, \ldots, E
\end{array}
$$

Assim, o modelo (1)-(9) substituindo a restrição (6) por (12), (13), (14) é um modelo linear, cuja restrição de capacidade da serra considera que cada ciclo da serra é constituído por um único padrão de corte, como queríamos. Este modelo será denotado por RPIDCM.

Com este novo modelo, tende-se a obter resultados piores quando comparados ao modelo original PIDCM, isto é, piores valores objetivo, já que serão necessários mais ciclos da serra, consequêntemente uma maior capacidade é necessária, sofrendo alteração no plano de produção quando comparado ao modelo PIDCM, como pode ser visto no exemplo a seguir.

Exemplo 1. (Vanzela et al., 2013): Considere a produção de um único produto final $(F=1)$ em um horizonte de planejamento de 2 períodos $(T=2)$. Os itens são obtidos a partir do corte de objetos com $(2000 \times 1000)$ mm. O estoque inicial do produto final $\left(I_{f 0}\right)$ é 9 unidades. O custo de produção e estoque do produto final são respectivamente, 42 e 0,063 reis. O custo dos objetos foi considerado 80 e 100 reais para as espessuras de 15 e 18 mm respectivamente. A demanda deste produto final para cada período é respectivamente 10 e 5 unidades. A estrutura dos produtos é composta por um conjunto de 4 objetos $(P=4)$ divididas entre duas espessuras distintas $(E=2)$, como pode ser visto na tabela 1 . Consideramos os parâmetros de estimativa de perda (tx) e estoque de segurança (tseg), respectivamente 1,15 e 0,6. Na formulação, para a capacidade da serra atribuímos 11 e 9 ciclos de serra respectivamente para cada período. Para este exemplo consideramos os padrões de corte homogêneos e como solver utilizamos o CPLEX para resolver a instância dos modelos PIDCM e RPIDCM gerados com estes dados.

Tabela 1. Estrutura do Produto $\left(q_{p f}^{e}\right)$ e Dimensões do item $(l \times w)$

\begin{tabular}{c|ccc|c|ccc}
\hline \multicolumn{4}{c|}{ Espessura 15mm } & \multicolumn{4}{c}{ Espessura 18mm } \\
\hline Item & Quantidade de itens & $l(\mathrm{~mm})$ & $w(\mathrm{~mm})$ & Item & Quantidade de itens & $l(\mathrm{~mm})$ & $w(\mathrm{~mm})$ \\
1 & 3 & 680 & 198 & 3 & 4 & 630 & 340 \\
2 & 4 & 760 & 60 & 4 & 5 & 1120 & 450 \\
\hline
\end{tabular}

As Tabelas 2, 3 e 4 mostram os resultados dos dois modelos. Como era de se esperar, a função objetivo do modelo RPIDCM mostrou-se pior quando comparada ao modelo PIDCM. Nota-se algumas mudanças na quantidade de objetos cortados de uma formulação para outra o que esta diretamente relacionado a nova restrição de capacidade da serra.

Tabela 2. Função objetivo

\begin{tabular}{c|c}
\hline & Função Objetivo \\
\hline PIDCM & 5993,88 \\
\hline RPIDCM & 5999,67 \\
\hline
\end{tabular}


Tabela 3. Número de objetos cortados para cada formulação

\begin{tabular}{c|c|c}
\hline Variável $y_{j t}^{e}$ & PIDCM & RPIDCM \\
\hline$y_{11}^{1}$ & 3 & 3 \\
$y_{11}^{2}$ & 0 & 0 \\
$y_{21}^{1}$ & 1 & 2 \\
$y_{21}^{2}$ & 0 & 0 \\
$y_{31}^{1}$ & 0 & 0 \\
$y_{31}^{2}$ & 5 & 6 \\
$y_{41}^{1}$ & 0 & 0 \\
$y_{41}^{2}$ & 19 & 21 \\
$y_{12}^{1}$ & 2 & 2 \\
$y_{12}^{2}$ & 0 & 0 \\
$y_{22}^{1}$ & 1 & 0 \\
$y_{22}^{2}$ & 0 & 0 \\
$y_{32}^{1}$ & 0 & 0 \\
$y_{32}^{2}$ & 5 & 4 \\
$y_{42}^{1}$ & 0 & 0 \\
$y_{42}^{2}$ & 19 & 17 \\
\hline
\end{tabular}

Tabela 4. Contagem de ciclos $\left(\frac{y_{j t}^{e}}{c a p^{e}}\right)$

\begin{tabular}{l|c|c|c|c|c|c|c|c}
\hline Período $\mathrm{t}=1$ & \multicolumn{7}{c}{ Contagem dos Ciclos } \\
\hline \hline PIDCM & 0,75 & 0 & 0,25 & 0 & 0 & 1,67 & 0 & 6,33 \\
RPIDCM & 0,75 & 0 & 0,5 & 0 & 0 & 2 & 0 & 7 \\
$z_{j t}^{e}$ & 1 & 0 & 1 & 0 & 0 & 2 & 0 & 7 \\
\hline Período t $=2$ & \multicolumn{7}{|c}{ Contagem dos Ciclos } \\
\hline \hline PIDCM & 0,5 & 0 & 0,25 & 0 & 0 & 1,67 & 0 & 6,33 \\
RPIDCM & 0,5 & 0 & 0 & 0 & 0 & 1,33 & 0 & 5,67 \\
$z_{j t}^{e}$ & 1 & 0 & 0 & 0 & 0 & 2 & 0 & 6 \\
\hline
\end{tabular}

\section{CONCLUSÕES E PERSPECTIVAS FUTURAS}

Neste trabalho, abordamos a formulação proposta por Vanzela et al. (2013) e propomos uma reformulação para a restrição de ciclos da serra. A principal motivação para este estudo, é o fato da restrição original constituir uma relaxação para a restrição real de ciclos da serra, em que padrões de corte diferentes não podem compartilhar o mesmo ciclo da serra. No entanto a reformulação proposta é não linear, deste modo uma estratégia de linearização foi aplicada para torna-la linear.

Para avaliar as consequências desta nova restrição no modelo, pretende-se estender a técnica de geração de colunas utilizada por Vanzela et al. (2013) e aplicá-la para resolver o modelo reformulado (RPIDCM) usando os dados reais da Fabrica L.

\section{AGRADECIMENTOS}

Esta pesquisa contou com o apoio financeiro da FAPESP (CEPID e Projeto Temático).

\section{REFERÊNCIAS}

Alem, D. J., e Morabito, R. 2012. Production planning in furniture settings via robust optimization. Computers \& Operations Research, 39, 139 - 150.

Farley, A. A. 1988. Mathematical programming models for cutting-stock problems in the clothing industry. The Journal of Operational Research Society, 39, 41-53.

Figueiredo, A., e Rangel, S. 2008. Geração de padrões de corte produtivos para a indústria de móveis. Anais do XXXVIII Simpósio Brasileiro de Pesquisa Operacional - SBPO, (pp. 1626 - 1636).

Ghidini, C. T. L. S., e Arenales, M. N. 2009. Otimização de processos acoplados na indústria de móveis: dimensionamento de lotes e corte de estoque.

Gramani, M., França, P. M., e Arenales, M. N. 2011. A linear optimization approach to the combined production planning mode. Journal of the Franklin Institute, 348, 1523 - 1536. 
Gramani, M. C. N., e França, P. M. 2006. The combined cutting stock and lot-sizing problem in industrial processes. European Journal of Operational Research, 174, 509 - 521.

Hendry, L. C., Fok, K. K., e Shek, K. W. 1996. A cutting stock and scheduling problem in the copper industry. The Journal of the Operational Research Society, 47, 38 - 47.

Melega, G. M., Araujo, S. A. d., e Rangel, S. 2014. Uma formulação não linear para a restrição de ciclos da serra no problema integrado de dimensionamento de lotes e corte de estoque. Livro de resumos do XVI Oficina Nacional de Problemas de Corte e Empacotamento, Planejamento e Programação da Produção e Correlatos - ONPCE.

Mosquera, G., e Rangel, S. 2007. Redução de ciclos da serra no problema de corte de estoque bidimensional na indústria de móveis. Anais do XXX - Congresso Nacional de Matemática Pura e Aplicada - CNMAC.

Nonas, S. L., e Thorstenson, A. 2008. Solving a combined cutting-stock and lot-sizing problem with a column generating procedure. Computers \& operations research, 35.

Poltroniere, S. C., Poldi, K. C., Toledo, F. M. B., e Arenales, M. N. 2008. A coupling cutting stock-lot sizing problem in the paper industry. Annals of Operations Research, 157.

Ranck JR., R. 2008. Desenvolvimento de alguns métodos de solução para o problema de redução de ciclos da serra. Dissertação de Mestrado, Dissertação de Mestrado, INPE.

Santos, S., Araujo, S. A., e Rangel, S. 2011. Integrated cutting machine programming and lot sizing in furniture industry. Pesquisa Operacional para o Desenvolvimento, 3, 249 - 266.

Toscano, A., Rangel, S., e Yanasse, H. H. 2013. A heuristic approach to minimize the number of saw cycles in small-scale furniture factories. Relatório Técnico, DMAp - UNESP.

Vanzela, M., Rangel, S., e de Araujo, S. 2013. A study of the integrated lot sizing and cutting stock problem for furniture production. Relatório Técnico, DMAp - UNESP.

Yanasse, H. H. 2008. A note on the minimization of the number of cutting cycles problem. Livro de resumos do XI Simpósio de Pesquisa Operacional e Logística da Marinha - SPOLM.

Yanasse, H. H., Zinober, A. S. I., e Harris, R. G. 1991. Two-dimensional cutting stock with multiple stock sizes. The Journal of the Operational Research Society, 42, $673-683$.

\title{
7. SOBRE RESPONSABILIDADE AUTORAL E O USO DE IDIOMA PORTUGUÊS OU ESPANHOL
}

\section{RESPONSABILIDADE AUTORAL}

“Os autores são os únicos responsáveis pelo conteúdo deste trabalho”.

\section{INTEGRATED LOT SIZING AND CUTTING STOCK PROBLEM WITH SAW CYCLES CONSTRAINS}

\author{
Gislaine M. Melega, gislainemelega@gmail. com ${ }^{1}$ \\ Matheus Vanzela, matheusvanzela@gmail.com ${ }^{1}$ \\ Silvio A. de Araujo, saraujo@ibilce.unesp.br.tres ${ }^{1}$ \\ Socorro Rangel, socorro@ibilce. unesp.br. quatro ${ }^{1}$ \\ ${ }^{1}$ Unesp - Ibilce, Departamento de Matemática Aplicada, São José do Rio Preto, SP
}

Resumo. This paper addresses the integrated lot sizing problem and cutting stock problem. The goal of integrated problem is to capture the interdependencies between the decisions of these problems in order to reduce raw material usage and costs the production process. We discuss a literature model that presents a study of case in a small furniture factory and consider in their model constrains to control the number of saw cycles. We propose an extension of this set of constrains, that results in a nonlinear model. A linearization strategy is applied to these constrains, thereby obtaining a linear model. The aim of this new model is extending the column generation technique which was applied to the original model and discuss the results.

Palavras-chave: Lot Sizing Problem, Cutting Stock Problem, Integrated Problem, Saw Cycles. 